2003

\title{
Strong ion-sound parametric turbulence and anomalous anisotropic plasma heating in helicon plasma sources
}

\author{
V.S. Mikhailenko \\ K. N. Stepanov \\ E. E. Scime
}

Follow this and additional works at: https://researchrepository.wvu.edu/faculty_publications

\section{Digital Commons Citation}

Mikhailenko, V. S.; Stepanov, K. N.; and Scime, E. E., "Strong ion-sound parametric turbulence and anomalous anisotropic plasma heating in helicon plasma sources" (2003). Faculty Scholarship. 350.

https://researchrepository.wvu.edu/faculty_publications/350 


\title{
Strong ion-sound parametric turbulence and anomalous anisotropic plasma heating in helicon plasma sources
}

\author{
V. S. Mikhailenko and K. N. Stepanov \\ Kharkiv National University, 31 Kurchatov Prosp, 61108 Kharkiv, Ukraine \\ E. E. Scime \\ Physics Department, West Virginia University, Morgantown, West Virginia 26506
}

(Received 14 October 2002; accepted 3 March 2003)

\begin{abstract}
Experimentally observed anisotropic ion heating in helicon plasma sources may result from the interaction of ions with ion-sound turbulence. The ion-sound turbulence develops due to excitation of the short scale, kinetic, parametric ion-sound instability. From the quasilinear equation for the ion distribution function, which includes the induced scattering of ion-sound waves by ions, the turbulent heating rate is determined. This anomalous ion heating is predominantly across the magnetic field and can lead to strong turbulence effects, such as the collective statistical effect of ion-sound turbulence on individual ion orbits (resonance broadening). Resonance broadening is a strong turbulence saturation mechanism for ion-sound turbulence. The energy density of the ion-sound turbulence in the saturated state, the ion and electron heating rates, as well as the effective collision frequency arising from the anomalous absorption of the helicon wave, are estimated and compared to experimental data. (C) 2003 American Institute of Physics.
\end{abstract}

[DOI: $10.1063 / 1.1569487]$

\section{INTRODUCTION}

Helicon plasma sources efficiently produce high-density plasmas for moderate rf input powers at frequencies of 8-27 MHz. Since Boswell's initial helicon wave experiments, ${ }^{1}$ the mechanisms responsible for the high rf absorption efficiency of helicon sources and anomalously strong electron heating have been the subject of both theoretical and experimental investigations. Experimental studies have shown that collisional damping of helicon waves as well as electron Landau damping is insufficient to explain the high rf absorption efficiency and electron heating observed in helicon sources. ${ }^{2}$ It has been suggested that helicon waves deposit their energy into the plasma by coupling their energy to TrivelpieceGould waves at the plasma boundary, which are then rapidly absorbed. ${ }^{3-5}$

It was suggested in Ref. 6 that another mechanism: excitation of the kinetic, parametric, ion-sound instability and the subsequent turbulent (quasilinear) heating of electrons in the fields of the ion-sound turbulence along the confining magnetic field may be responsible for electron heating and helicon energy absorption in helicon plasma sources. Recently, that theoretical suggestion received strong experimental support. Although the theoretically predicted wavelengths for the parametric ion sound turbulence are too small to be measured with probes, somewhat longer wavelength, lowfrequency fluctuations in helicon sources have been detected by a cross-correlation enhanced-scattering diagnostic ${ }^{7,8}$ and with fixed probe pairs ${ }^{9,10}$ (see also Ref. 11). The dispersion relations deduced from the measured frequencies and wavelengths were consistent with turbulent ion-acoustic fluctuations ${ }^{7}$ and the helicon wave damping was found to increase with fluctuation amplitude. ${ }^{8}$ Electron heating, corresponding to periods of increased fluctuation amplitudes, was measured by both emission spectroscopy and rf compensated Langmuir probes. ${ }^{8}$ These measurements demonstrated, for the first time, that the anomalous rf power absorption as well as electron heating in helicon discharges is associated with excitation of small-scale, ion-sound-like, fluctuations.

In addition to electron heating, anisotropic $\left(T_{i \perp}>T_{i \|}\right)$ ion heating has also been observed in helicon sources. ${ }^{12,13}$ Careful analysis demonstrated that collisional equilibration with electrons could not be responsible for the magnitude of ion heating observed in helicon sources. ${ }^{12}$ Recent measurements of ion temperatures tens of centimeters downstream of a helicon antenna are consistent with damping of the slow, or "Trivelpiece-Gould," wave on ions at the edge of the helicon source when the rf frequency equals the local lower hybrid frequency. ${ }^{9,13}$ Closer to the antenna, the perpendicular ion temperatures appear to be correlated with the amplitude of low-frequency, ion acoustic waves that are parametrically driven by the rf pump wave., ${ }^{9,10}$ In this paper, we show that anisotropic ion heating near the rf antenna may result from turbulent ion heating in the wave fields of ion-sound parametric turbulence. We find that in helicon source experiments, the ion-sound parametric turbulence is predicted to be in the strong turbulence regime. Therefore, the dynamics of ions and electrons in such sources is strongly nonlinear and is determined by their "collisions" with the ion-sound fluctuations.

The remainder of this paper is organized as follows. In Sec. II we give a brief description of weak ion-sound parametric turbulence excited due to development of the ionsound kinetic parametric instability and describe the processes of electron turbulent heating and anomalous absorption of the helicon wave. In Sec. III, through examination of the nonlinear quasilinear equation, which includes 
the induced scattering of ion-sound waves on ions, we show that the ion-sound parametric turbulence can result in anisotropic ion heating. In Sec. IV we show that the initially weak turbulence is followed by the development of strong turbulence during which ion scattering by ion sound turbulence results in enhanced ion Landau damping. This effect leads to the saturation of ion-sound turbulence and to perpendicular ion heating. The magnitude of the strong ion-sound turbulence, the ion-heating rate and the effective collision frequency for the saturated, strongly turbulent state are estimated. Some concluding remarks are then given in Sec. V.

\section{THE ION-SOUND KINETIC PARAMETRIC INSTABILITY, TURBULENCE, ELECTRON HEATING, AND HELICON WAVE ABSORPTION}

The action of a strong electromagnetic wave on a plasma results in relative oscillatory motion of plasma components that can drive a variety of parametric instabilities. The linear stage of these instabilities is governed by the following equation for the Fourier transformation of the perturbed electrostatic potential $\varphi(\mathbf{k}, \omega)$ :

$$
\begin{aligned}
(1+ & \left.\delta \varepsilon_{i}(\mathbf{k}, \omega)\right) \varphi(\mathbf{k}, \omega)+\sum_{n=-\infty}^{\infty} \sum_{p=-\infty}^{\infty} J_{p}(a) J_{p+n}(a) \\
& \times e^{i n \delta} \delta \varepsilon_{e}\left(\mathbf{k}-p \mathbf{k}_{0}, \omega-p \omega_{0}\right) \varphi\left(\mathbf{k}+n \mathbf{k}_{0}, \omega+n \omega_{0}\right)=0
\end{aligned}
$$

where $\delta \varepsilon_{i, e}(\mathbf{k}, \omega)$ is the ion (electron) dielectric permittivity, $J_{p}(a)$ is the Bessel function with argument $a=a_{e}$ $\approx c\left(k_{x}^{2} E_{0 y}^{2}+k_{0 y}^{2} E_{0 x}^{2}\right)^{1 / 2} / \omega_{0} B_{0}, \quad \delta=\delta_{e} \approx \tan ^{-1}\left(k_{x} E_{0 y} / k_{y} E_{0 x}\right)$ and $E_{0 x}$ and $E_{0 y}$ are radial and azimuthal components, respectively, of the helicon or Trivelpiece-Gould modes electric field $\mathbf{E}_{0}$.

The kinetic parametric ion-sound instability is excited in nonisothermal plasmas with hot electrons and cold ions $\left(T_{e} \gg T_{i}\right.$ ) when the amplitude of the electron oscillatory velocity $u$ in the field $\mathbf{E}_{0}=\mathbf{e}_{x} E_{0 x} \cos \left(\omega_{0} t-\mathbf{k}_{0} \mathbf{r}\right)+\mathbf{e}_{y} E_{0 y} \sin \left(\omega_{0} t\right.$ $\left.-\mathbf{k}_{0} \mathbf{r}\right)$ of the helicon wave exceeds the ion-sound velocity $\mathrm{v}_{s}$. For short wavelength ion-sound waves with $k_{\perp} \rho_{e} \gg 1$, where $\rho_{e}$ is the electron Larmor radius, Eq. (1) becomes

$$
\begin{gathered}
{\left[1-\frac{\omega_{p i}^{2}}{\omega^{2}}+\frac{1}{k^{2} r_{D e}^{2}}+i \frac{\sqrt{\pi}}{k^{2} r_{D e}^{2}} I_{0}\left(k^{2} \rho_{e}^{2}\right) e^{-k^{2} \rho_{e}^{2}}\right.} \\
\left.\times \sum_{p=-\infty}^{\infty} J_{p}^{2}(a) z_{e}^{p} W\left(z_{e}^{(p)}\right)\right] \varphi(\mathbf{k}, \omega)=0 .
\end{gathered}
$$

Here $W\left(z_{e}^{(p)}\right)$ is the plasma dispersion function with the argument $z_{e}^{(p)}=\left(\omega-p \omega_{0}\right) / \sqrt{2}\left|k_{\|}-p k_{0 \|}\right| \mathrm{v}_{T e}, k_{\|}$and $k_{0 \|}$ are the components of the wave numbers $\mathbf{k}$ and $\mathbf{k}_{0}$ ( $\mathrm{rf}$ driving wave) along the magnetic field $\mathbf{B}_{0}, I_{0}$ is the modified Bessel function, and $r_{D e}$ is Debye length. It follows from Eq. (2) that the frequency $\omega(\mathbf{k})$ and growth rate $\gamma(\mathbf{k})$ of this instability for short ion-sound waves, are ${ }^{6}$

$$
\begin{aligned}
\omega(\mathbf{k}) \equiv & \omega_{s}(\mathbf{k})=k \mathrm{v}_{s}\left(1+k^{2} r_{D e}^{2}\right)^{-1 / 2}, \\
\gamma(\mathbf{k})= & \gamma_{e}(\mathbf{k})=\sum_{p=-\infty}^{\infty} \gamma_{e p}(\mathbf{k}) \\
= & -\frac{\omega_{s}(\mathbf{k})}{2^{3 / 2} k_{\perp} \rho_{e}\left(1+k^{2} r_{D e}^{2}\right)} \sum_{p=-\infty}^{\infty} J_{p}^{2}(a) z_{e 0}^{(p)} \\
& \times \exp \left(-\left(z_{e 0}^{(p)}\right)^{2}\right),
\end{aligned}
$$

where $z_{e 0}^{(p)}=\left(\omega_{s}(k)-p \omega_{0}\right) / \sqrt{2}\left|k_{\| p}\right| \mathrm{v}_{T e}$, and $k_{\| p}=k_{\|}-p k_{0 \|}$. In the case of strong ellipticity of the pump wave, when $E_{0 x}>E_{0 y}$, the growth rate is a maximum with

$$
\gamma_{\max } \sim 0.05\left(\omega_{c e} \omega_{c i}\right)^{1 / 2}\left(1+k^{2} r_{D e}^{2}\right)^{-3 / 2}
$$

for ion-sound waves with frequency $\omega_{s} \leqslant \omega_{0}$ and an anisotropic wave spectrum such that

$$
k_{y}\left|\frac{E_{0 x}}{E_{0 y}}\right| \geqslant k_{x} \sim \frac{\omega_{0}}{\mathrm{v}_{s}} \gg k_{y} \sim \frac{\omega_{0}}{u} a,
$$

where $u \approx c E_{0 x} / B_{0} \gg a \mathrm{v}_{s}$.

For ion sound waves, the greatest contribution to the growth rate arising from the sum over the Bessel function terms in Eq. (4) is from the term with $p=1$, which is a maximum for $a \approx 1.8-2.2$. Contributions to $\gamma_{e}$ by the other terms with $p \neq 1$ are much smaller. Note that the ions are treated as unmagnetized $\left(2 \pi \gamma_{e} \gg \omega_{c i}, k_{\perp} \rho_{i} \gg 1\right)$. Equation (5) was obtained without including ion Landau damping of the ionsound waves, $\gamma_{i}$, or collisional damping due to ion viscosity, $\gamma_{\mathrm{v}}$. When these effects are included, the total growth rate is given by

$$
\gamma=\gamma_{e}-\gamma_{i}-\gamma_{\mathrm{v}}
$$

where

$$
\begin{aligned}
\gamma_{i} & =\frac{\sqrt{\pi}}{2} \omega(k) \frac{T_{e}}{T_{i}} \frac{z_{i} e^{-z_{i}^{2}}}{\left(1+k^{2} r_{D e}^{2}\right)} \\
\gamma_{\mathrm{v}} & =\frac{4}{5} \nu_{i i} \frac{T_{i}}{T_{e}}\left(1+k^{2} r_{D e}^{2}\right)
\end{aligned}
$$

and

$$
\begin{aligned}
& z_{i}=\sqrt{\frac{T_{e}}{2 T_{i}}}\left(1+k^{2} r_{D e}^{2}\right)^{-1 / 2}, \\
& \nu_{i i}=\frac{4}{3}\left(\frac{\pi}{m_{i}}\right)^{1 / 2} \frac{e_{i}^{4} n_{0 i}}{T_{i}^{3 / 2}} L_{i}, \quad L_{i}=\ln \frac{T_{i}^{3 / 2}}{e_{i}^{3}\left(4 \pi n_{0 i}\right)^{1 / 2}} .
\end{aligned}
$$

From Eq. (4) through Eq. (6), it follows that for $k \sim \omega_{0} / \mathrm{v}_{s}$ we have $k \rho_{e} \sim \omega_{0} \mathrm{v}_{\mathrm{Te}} / \mathrm{v}_{\mathrm{s}} \omega_{c e} \sim \omega_{0} / \sqrt{\omega_{c e} \omega_{c i}}$ for the most unstable ion-sound waves. In the case where the pumping frequency, $\omega_{0}$, is much larger than the lower hybrid frequency $\sqrt{\omega_{c i} \omega_{c e}}$, the most unstable ion-sound waves have short wavelengths with $k \rho_{e} \gg 1$, consistent with the approximations used to obtain Eq. (5). However, the form of Eq. (4) suggests that the value of $\left|\gamma_{\max }(k) / \omega(k)\right|$ for the most unstable ion-sound waves should increase for $k \rho_{e} \sim 1$. These two conditions, $k \rho_{e} \sim \omega_{0} / \sqrt{\omega_{c e} \omega_{c i}}$ and $k \rho_{e} \sim 1$, are satisfied when 
the frequency of the rf pump wave is of the order of the lower hybrid frequency, $\omega_{0}=\sqrt{\omega_{c i} \omega_{c e}}$ in a high density plasma, i.e., $\omega_{p i} \gg \omega_{c i}$.

Since the analytically derived Eq. (4) cannot be used in the case of $k \rho_{e} \sim 1$, a complete numerical solution of Eq. (1) is required. The numerical solution of Eq. (1), including Landau damping for a helicon wave frequency of $\omega_{o}$ $\approx 0.6 \sqrt{\omega_{c i} \omega_{c e}}$, for $T_{e} / T_{i}=20$ and $u / \mathrm{v}_{s}=5$ yields

$$
\gamma_{\max } \approx 0.3 \sqrt{\omega_{\mathrm{ce}} \omega_{c i}} \text { and } \operatorname{Re} \omega(k) \approx 0.9 \sqrt{\omega_{\mathrm{ce}} \omega_{c i}},
$$

for the growth rate and real frequency of the kinetic parametric instability ${ }^{15}$ at $k \rho_{e} \approx 0.75$. This value of $\left|\gamma_{\max } / \operatorname{Re} \omega(k)\right|$ is six times larger than the value given by Eq. (5) for the same plasma parameters but in the limit $k \rho_{e} \gg 1$.

Saturation of the kinetic parametric ion sound instability, electron and ion heating, and helicon wave absorption are all governed by the development of weak-nonlinear and quasilinear processes ${ }^{6}$ as well as the development of strongly nonlinear processes such as the collective statistical effect of ion-sound turbulence on individual ion orbits (resonance broadening). The induced scattering of the ion-sound waves by ions was determined in Ref. 6 as being primarily a weakly nonlinear process that can lead to saturation of kinetic parametric ion sound instability. The energy density $W$ $=\int d \mathbf{k} W(\mathbf{k})=\int d \mathbf{k} k^{2} \omega(\mathbf{k}) I(\mathbf{k}) \partial \operatorname{Re} \varepsilon(\mathbf{k}) / 4 \pi \partial \omega(\mathbf{k})$ of the ion sound parametric turbulence in the saturated state was determined in Ref. 6 from the equation $\gamma+\Gamma_{i}=0$, where $\gamma$ is given by Eq. (7) and $\Gamma_{i}$ is the nonlinear damping rate arising from the induced scattering of ion-sound waves by the ions, ${ }^{16}$

$$
\begin{aligned}
\Gamma_{i}(\mathbf{k})= & \frac{2 \pi e^{2}}{m_{i}^{2}} \frac{T_{i}}{T_{e}} \frac{\left(1+k^{2} r_{D e}^{2}\right)^{5 / 2}}{k^{5} v_{s}^{3}} \frac{\partial}{\partial k} \int d \mathbf{k}_{1} \\
& \times I\left(\mathbf{k}_{1}\right)\left(\mathbf{k} \cdot \mathbf{k}_{1}\right)^{2}\left(\mathbf{k} \times \mathbf{k}_{1}\right)^{2} \delta\left(k-k_{1}\right),
\end{aligned}
$$

where the spectral intensity $I(\mathbf{k})$ is determined by the relation $^{16}$

$$
\begin{aligned}
\left\langle\varphi(\mathbf{k}, \omega) \varphi\left(\mathbf{k}^{\prime}, \omega^{\prime}\right)\right\rangle= & I(\mathbf{k}) \delta(\omega-\omega(\mathbf{k})) \delta\left(\omega-\omega^{\prime}\right) \delta(\mathbf{k} \\
& \left.-\mathbf{k}^{\prime}\right)
\end{aligned}
$$

and $\langle\ldots\rangle$ means the averaging over the initial phases of the perturbed potential $\varphi(\mathbf{k}, \omega)$. As was shown in Ref. 17,

$$
W \sim n_{0 e} T_{e}\left(T_{e} / T_{i}\right)\left(8 \gamma / \pi k \mathrm{v}_{s}\right) .
$$

The factor of 8 in Eq. (12) is an approximation for the integration over angles in wave number space according to Eq. (11) (this multiplicative factor is missing in Ref. 6).

The scattering of electrons by the ion-sound waves can also lead to electron heating and the electron heating rate as calculated in Refs. 6 and 17 from the quasilinear equation for the electron distribution function is

$$
\frac{3}{2} \frac{\partial T_{e}}{\partial t}=\frac{T_{e \|}}{\tau_{e \|}}
$$

where

$$
\begin{aligned}
\frac{1}{\tau_{e \|}} & =\sum_{p=-\infty}^{\infty} \int d \mathbf{k} \frac{I(\mathbf{k})}{n_{0 e} T_{e}}\left(\frac{\partial \varepsilon_{i}}{\partial \omega(\mathbf{k})}\right) \frac{\left(p \omega_{0}-\omega(\mathbf{k})\right)}{\omega(\mathbf{k})} \gamma_{p e}(\mathbf{k}) \\
& \sim \gamma_{e} \frac{W}{n_{0 e} T_{e}} .
\end{aligned}
$$

For the level $W$ given by Eq. (12) we obtain the estimate

$$
\frac{1}{\tau_{e \|}} \sim\left(p \frac{\omega_{0}}{\omega_{s}}-1\right) \frac{8 \gamma \gamma_{e}}{\pi k \mathrm{v}_{\mathrm{s}}} .
$$

In obtaining Eq. (13) we assumed that $\tau_{e \|} \nu_{e e} \gg 1$, i.e., during the heating time $\tau_{e \|}$, numerous binary electron collisions take place and an isotropic Maxwellian electron distribution with $T_{e \|} \approx T_{e}$ evolves.

The helicon wave damping due to the excitation of ionsound turbulence and the resultant turbulent electron heating can be estimated using the effective collision frequency due to electron scattering on the ion-sound waves. The effective collision frequency is obtained from the energy balance equation

$$
2 \nu_{\text {eff }} \frac{k_{0}^{2} c^{2}}{\omega_{p e}^{2}} W_{\text {helicon }} \approx n_{0 e} \frac{d T_{e}}{d t}=\frac{n_{0 e} T_{e \|}}{\tau_{e \|}},
$$

where $\mathbf{k}_{0}$ and $W_{\text {helicon }}$ are the helicon wave number and helicon energy density, respectively,

$$
\begin{aligned}
& k_{0}=\frac{\omega_{p e}^{2} \omega_{0}}{\omega_{c e} c^{2} k_{0 \|}}, \\
& W_{0} \approx \frac{1}{16 \pi}|\widetilde{B}|^{2} \approx \frac{m_{e} n_{0 e} u^{2}}{2} \frac{\omega_{c e}}{\omega_{0}} \frac{k_{0 \|}}{k_{0}},
\end{aligned}
$$

$\widetilde{B}$ is the helicon wave magnetic field strength, and $u$ $=c E_{0} / B_{0}$. Combining Eqs. (14), (16) and (17), $\nu_{\text {eff }}$ is approximately $^{6}$

$$
\nu_{\mathrm{eff}} \sim \gamma_{e} \frac{W}{2 W_{0}} \frac{\omega_{c e}}{\omega_{0}} \frac{k_{0 \|}}{k_{0}} \sim \gamma_{e} \frac{W}{n_{0 e} T_{e}} \frac{\mathrm{v}_{T \mathrm{e}}^{2}}{u^{2}} .
$$

For the energy density given by Eq. (12) we obtain the estimate

$$
\nu_{\mathrm{eff}} \sim 16 \gamma_{e} \frac{T_{e}}{T_{i}} \frac{\gamma}{\omega_{s}} \frac{\mathrm{v}_{\mathrm{Te}}^{2}}{\mathrm{u}^{2}} .
$$

Another source for the excitation of the parametric ionsound turbulence is the Trivelpiece-Gould (TG) wave, which is excited due to the mode conversion from the helicon wave near the plasma surface. The TG mode is a short wavelength wave along the radial coordinate with $\operatorname{Re} k_{0 x}$ $=\left(\omega_{c e} / \omega_{0}\right) k_{0 \|}$ and is strongly damped with $\operatorname{Im} k_{0 x}$ $=-\operatorname{Re} k_{0 x}\left(\nu_{e i} / \omega\right)$. The optimal conditions for the maximum growth rate, $\gamma_{e}$, given by Eq. (6) for an anisotropic pump wave $\left(E_{\mathrm{TG}, y} \ll E_{\mathrm{TG}, x}\right)$ and $k_{y} \sim \omega_{0} a / u_{e}, k_{x} \sim \omega_{0} / \mathrm{v}_{s}$, remain valid in the case of the TG pumping wave. For the TG mode we have ${ }^{3} \quad E_{\mathrm{TG}, y} \approx 0$ and $E_{T G, x}$ $=\left(\omega_{c e} / \omega_{0}\right) E_{\text {Helicon, } y}$. For the conditions of, for example, the Chen experiment $\left[B_{0}=800 \mathrm{G}, E_{y, \text { Helicon }}=6 \mathrm{~V} / \mathrm{cm}\right.$ and $\left.\omega_{0}=1.7 \times 10^{8} \mathrm{~s}^{-1}(f=27 \mathrm{Mhz})\right]$ we have $E_{\mathrm{TG}, x}=500 \mathrm{~V} / \mathrm{cm}$, $u \sim 5 \times 10^{7} \mathrm{~cm} / \mathrm{s}$, and for the wave number of the most un- 
stable ion-sound waves, $k_{y} \sim 1 \mathrm{~cm}^{-1}$ and $k_{x} \sim 10^{2} \mathrm{~cm}^{-1}$. Therefore, the azimuthal wavelengths of ion-sound waves excited by the TG wave should be large enough to be measured in helicon source experiments. The effective collision frequency for the absorption of the TG wave is determined from the equation

$$
2 \nu_{\mathrm{eff}} W_{\mathrm{TG}}=\nu_{\mathrm{eff}} \frac{n_{0 e} m_{e} u^{2}}{2}=n_{0 e} \frac{d T_{e}}{d t}=n_{0 e} \frac{T_{e \|}}{\tau_{e \|}},
$$

from which we obtain the following estimate for $\nu_{\text {eff }}$

$$
\nu_{\mathrm{eff}} \sim \frac{1}{\tau_{h}} \frac{\mathrm{v}_{T e}^{2}}{u^{2}} \sim \gamma_{e} \frac{W}{n_{0 e} T_{e}} \frac{\mathrm{v}_{T \mathrm{e}}^{2}}{u^{2}} .
$$

Because the velocity $u$ in a TG wave is much larger than in a helicon wave, Eq. (21) [analytically the same as Eq. (18)] yields a much smaller magnitude of $\nu_{\text {eff }}$ for the TG wave than for the helicon wave. For a TG wave in a helicon source operating at $T_{e} \sim 4 \mathrm{eV}$ and at the conditions of Chen's experiment, ${ }^{18} \nu_{\text {eff }}$ is also smaller than the ion-electron collision frequency, $\nu_{e i}$. Therefore, the absorption of the TG wave remains predominantly collisional. The long wavelength ion-sound waves observed in recent experiments ${ }^{9-11}$ may be parametrically excited by the TG mode, but these easily detected long wavelength ion-sound waves are not responsible for the anomalous plasma heating and absorption of the TG wave. Their existence, however, can be considered as indirect evidence that short wavelength ion-sound waves parametrically driven by the helicon wave, which are excited at the same conditions and are responsible for the anomalous electron heating and anomalous absorption of the helicon wave, may also exist in the plasma.

\section{TURUBLENT ION HEATING}

It is commonly suggested that in helicon sources, the ions are heated isotropically by electron-ion collisions. The experimental discovery of the anisotropic ion heating predominantly across the magnetic field ${ }^{12}$ suggests that collisional equilibration with hotter electrons cannot be responsible for all the observed ion heating. Interaction of ions with anisotropic plasma turbulence may be the source of the anisotropic ion heating. Since the bulk ions are nonresonant with the ion-sound turbulence, it was suggested in Ref. 19 that induced scattering of ion sound waves by ions through nonlinear Landau damping might result in anisotropic ion heating. The quasilinear equation for the average ion distribution function $F_{i 0}$ in which the induced scattering of ionsound waves by ions is taken into account is ${ }^{20}$

$$
\begin{aligned}
\frac{\partial F_{i 0}}{\partial t}= & \pi \frac{e^{2}}{m_{i}^{2}} \int d \mathbf{k}\left(\mathbf{k} \cdot \frac{\partial}{\partial \mathbf{v}_{i}}\right) I(\mathbf{k}) \delta\left(\omega(\mathbf{k})-\mathbf{k} \cdot \mathbf{v}_{i}\right)\left(\mathbf{k} \cdot \frac{\partial F_{i}}{\partial \mathbf{v}_{i}}\right) \\
& +\frac{\pi}{m_{i}^{2} n_{i 0}^{2}} \int d \mathbf{k} \int d \mathbf{k}^{\prime} I(\mathbf{k}) I\left(\mathbf{k}^{\prime}\right) \\
& \times\left(\frac{\partial \varepsilon}{\partial \omega(\mathbf{k})}\right)^{-1}\left(\frac{\partial \varepsilon}{\partial \omega\left(\mathbf{k}^{\prime}\right)}\right)^{-1}\left(\left(\mathbf{k}-\mathbf{k}^{\prime}\right) \cdot \frac{\partial}{\partial \mathbf{v}_{i}}\right)\left(\mathbf{k} \cdot \mathbf{v}_{i}\right)
\end{aligned}
$$

$$
\begin{aligned}
& \times \frac{\left(\mathbf{k} \cdot \mathbf{k}^{\prime}\right)^{2}}{k^{2} k^{\prime 2}} \delta\left(\omega_{s}(\mathbf{k})-\omega_{s}\left(\mathbf{k}^{\prime}\right)-\left(\mathbf{k}-\mathbf{k}^{\prime}\right) \cdot \mathbf{v}_{i}\right)\left(\mathbf{k}-\mathbf{k}^{\prime}\right) \\
& \cdot \frac{\partial F_{i 0}}{\partial \mathbf{v}_{i}} .
\end{aligned}
$$

Because the wave vectors, $\mathbf{k}$, of the ion-sound waves excited by the kinetic parametric instability are directed primarily across the magnetic field, Eq. (22) predicts that the ions will be heated predominantly across the magnetic field. The predicted ion-heating rate is ${ }^{19}$

$$
\frac{\partial T_{i \perp}}{\partial t}=\frac{T_{e}}{\tau_{i \perp}},
$$

where

$$
\begin{aligned}
\frac{1}{\tau_{i \perp}}= & \frac{T_{i}}{2 m_{i}^{2} n_{i 0}^{2}} \int d \mathbf{k} \int d \mathbf{k}^{\prime} I(\mathbf{k}) I\left(\mathbf{k}^{\prime}\right) \\
& \times\left(\frac{\partial \varepsilon}{\partial \omega(\mathbf{k})}\right)^{-1}\left(\frac{\partial \varepsilon}{\partial \omega\left(\mathbf{k}^{\prime}\right)}\right)^{-1} \frac{\left(\mathbf{k} \cdot \mathbf{k}^{\prime}\right)^{2}}{k^{2} k^{\prime 2}} \\
& \times\left(\mathbf{k} \times \mathbf{k}^{\prime}\right)^{2} \delta\left(\omega_{s}(\mathbf{k})-\omega_{s}\left(\mathbf{k}^{\prime}\right)\right)
\end{aligned}
$$

and ion temperature anisotropy with $T_{i \perp}>T_{i \|}$ develops. For the saturated state of the turbulence due to the induced scattering of ion-sound waves on ions, we find that ${ }^{19}$

$$
\frac{1}{\tau_{i \perp}}=\int d \mathbf{k} \gamma(\mathbf{k}) \frac{W(\mathbf{k})}{n_{e o} T_{e}} \sim \frac{1}{\tau_{e \|}},
$$

and

$$
\frac{\partial T_{i \perp}}{\partial t} \sim \frac{\partial T_{e}}{\partial t}=\frac{T_{e}}{\tau_{e \|}} \sim \gamma_{e} \frac{W}{n_{e 0}} .
$$

\section{THE STRONG ION-SOUND TURBULENCE REGIME}

Ion heating leads to a reduced value of $T_{e} / T_{i}$ and therefore an increase in ion Landau damping, $\gamma_{i}$, of the ion-sound waves. Essentially, the entire process is a negative feedback cycle. The ion orbit perturbations induced by scattering from ion-sound waves leads to ion heating that then provides a saturation mechanism for the ion-sound instability through increased ion Landau damping.

However, collective statistical effects such as ion resonance broadening can also lead to saturation of the ion-sound instability. The energy density, $W$, of the ion-sound turbulence in the saturated state due to ion resonance broadening ${ }^{21}$ may be estimated from the equation $\gamma_{i}-\gamma_{e}=0$, where $\gamma_{e}$ is determined by Eq. (4) and $\gamma_{i}$ is the renormalized version of Eq. (8),

$$
\gamma_{i}=\left(\frac{\pi}{8}\right)^{1 / 2}\left(\frac{\omega_{s}}{k \mathrm{v}_{\mathrm{Ti}}}\right)^{3} \omega_{s} \exp \left(-\frac{\omega^{2}}{2 k^{2}\left(\mathrm{v}_{T i}^{2}+\tilde{\mathrm{v}}_{\mathrm{i}}^{2}\right)}\right) .
$$

In calculating the renormalized $\gamma_{i}$, we assume that the ion distribution is Maxwellian and account for the ion temperature increase due to the interaction of ions with ion-sound turbulence, 


$$
\begin{aligned}
\frac{\delta T_{i \perp}}{T_{i}} & \sim \frac{\left\langle\tilde{v}_{i}^{2}\right\rangle}{\mathrm{v}_{\mathrm{Ti}}^{2}} \sim \int d \mathbf{k} \frac{e^{2}\left\langle\widetilde{E}^{2}(\mathbf{k})\right\rangle}{m_{i}^{2} \omega_{s}^{2}} \frac{1}{\mathrm{v}_{\mathrm{Ti}}^{2}} \\
& \sim \int d \mathbf{k} k^{2} \frac{e^{2} I(\mathbf{k})}{m_{i}^{2} \omega_{s}^{2}} \frac{1}{\mathrm{v}_{\mathrm{Ti}}^{2}} \sim 2 \int d \mathbf{k} \frac{W(\mathbf{k})}{n_{i 0} T_{i}},
\end{aligned}
$$

where $\widetilde{\mathbf{E}}(\mathbf{k})$ is the electric field strength of the ion-sound wave with wave number $\mathbf{k}$ that is directed predominantly across the magnetic field $\left(k_{x}, k_{y} \gg k_{z}\right)$. From the equation $\gamma_{e}-\gamma_{i}=0$ we can obtain the energy density, $W$ of the ionsound turbulence in the saturated state due to ion resonance broadening:

$$
\frac{W}{n_{e 0} T_{e}} \sim \frac{1}{\ln \left[\frac{k \mathrm{v}_{s}}{\gamma_{e}}\left(\frac{\pi}{8}\right)^{1 / 2}\left(\frac{T_{e}}{T_{i}}\right)^{3 / 2}\right]}-\frac{2 T_{i}}{T_{e}} .
$$

For the growth rate given by Eq. (4), we have the estimate

$$
\frac{W}{n_{e 0} T_{e}} \sim \ln ^{-1}\left[\frac{\sqrt{2 e} k \rho_{e}}{J_{1}^{2}(a)}\left(\frac{T_{e}}{T_{i}}\right)^{3 / 2}\right]-\frac{2 T_{i}}{T_{e}} .
$$

Which saturation mechanism dominates is determined by comparing the energy densities in the saturated states due to induced scattering of ions on ion-sound waves, Eq. (12), and due to ion resonance broadening, Eq. (30):

$$
\begin{aligned}
& \frac{W(E q .(30))}{W(E q \cdot(12))} \\
& \quad \sim 10 \frac{T_{i}}{T_{e}} \frac{\omega_{0}}{\sqrt{\omega_{c i} \omega_{c e}}}\left\{\ln ^{-1}\left[8 \frac{\omega_{0}}{\sqrt{\omega_{c i} \omega_{c e}}}\left(\frac{T_{e}}{T_{i}}\right)^{3 / 2}\right]-\frac{2 T_{i}}{T_{e}}\right\} .
\end{aligned}
$$

According to Eq. (31), for high helicon rf wave frequencies, when $\omega_{0} \gg\left(T_{e} / T_{i}\right) \sqrt{\omega_{c e} \omega_{c i}}$, the induced scattering of ions on ion-sound waves may be the dominant saturation mechanism. In the case of low helicon rf wave frequencies, when $\omega_{0} \leqslant\left(T_{e} / T_{i}\right) \sqrt{\omega_{c e} \omega_{c i}}$, the dominant saturation mechanism is ion resonance broadening. This second condition is satisfied in the experiments of Refs. 12, 13, 14, 18. Therefore, it is reasonable to assume that the saturation level of ion-sound turbulence in these experiments results from ion resonance broadening and is given by Eq. (29). For the specific experimental conditions of Ref. 12 and Ref. 18, Eq. (30) yields $W / T_{e} n_{0 e} \sim 0.05$. For the same experimental conditions, Eq. (12), which calculates the saturation level of the ion-sound turbulence due to the induced scattering by ion sound waves, gives the unrealistically large value of $W / T_{e} n_{0 e} \sim 1$. It is important to note, that for the experimental parameters of Chen et al. ${ }^{18}\left(\gamma_{e} \sim 2 \times 10^{-6} \mathrm{~s}^{-1}\right.$ for $T_{e}=4 \mathrm{eV}$, $\tau_{e \|} \approx 3.3 \times 10^{-6} \mathrm{~s}, \quad T_{e} / T_{i}=20, \quad \nu_{e e} \approx 3.8 \times 10^{7} \mathrm{~s}^{-1}, \quad$ and $\left.W / n T_{e} \sim 0.05\right)$, we obtain $\tau_{e \|} \nu_{e e} \sim 20$, i.e., the electrons should thermalize rapidly and our assumption of $\tau_{e \|} \nu_{e e} \gg 1$ is justified. In this case, a multiplicative factor of $3 / 2$ appears in Eq. (13) and a Maxwell distribution for the bulk electrons should result from electron-electron collisions.

The effective frequency, $\nu_{\text {eff }}$, given by Eq. (17), for the energy density predicted by Eq. (29) is equal to

$$
\begin{aligned}
\nu_{\mathrm{eff}} & \sim \gamma_{e} \frac{W}{n_{0 e} T_{e}} \frac{\mathrm{v}_{T \mathrm{e}}^{2}}{u^{2}} \\
& \sim \gamma_{e} \frac{\mathrm{v}_{\mathrm{Te}}^{2}}{u^{2}}\left\{\ln ^{-1}\left[\frac{k \mathrm{v}_{s}}{\gamma_{e}}\left(\frac{\pi}{8}\right)^{1 / 2}\left(\frac{T_{e}}{T_{i}}\right)^{3 / 2}\right]-\frac{2 T_{i}}{T_{e}}\right\},
\end{aligned}
$$

and for the growth rate determined by Eq. (4) we have the estimate

$$
\nu_{\mathrm{eff}} \sim \gamma_{e} \frac{\mathrm{v}_{\mathrm{Te}}^{2}}{u^{2}}\left\{\ln ^{-1}\left[\frac{\sqrt{2 \pi e} k \rho_{e}}{J_{1}^{2}(a)}\left(\frac{T_{e}}{T_{i}}\right)^{3 / 2}\right]-\frac{2 T_{i}}{T_{e}}\right\} .
$$

For the Chen et al. ${ }^{18}$ experimental conditions, Eq. (33) gives $\nu_{\mathrm{eff}} \approx 3 \times 10^{8} \mathrm{~s}^{-1}$ and $\nu_{e i} \approx 3.8 \times 10^{7} \mathrm{~s}^{-1}$. Therefore, turbulent electron heating may be the primary helicon ionization mechanism in the Chen et al. experiment. The resulting helicon wave damping length along the magnetic field is $l_{T}$ $\sim \omega_{c e} / k_{0} \nu_{\text {eff }}$. For the experimental conditions of Chen et al. ${ }^{18}$ we obtain for $T_{e}=4 \mathrm{eV}$, that $l_{T} \leqslant 23 \mathrm{~cm}$, in agreement with the measurements reported in Ref. 18. The collisional damping length for these experimental parameters, $l_{c}$ $\sim \omega_{c e} / \nu_{e i} k_{0}$, is equal to $l_{c} \sim 2.2 \mathrm{~m}$. Thus, the helicon damping due to electron scattering by ion-sound waves is an order of magnitude larger than the collisional damping and is consistent with the measured damping length. ${ }^{18}$ It interesting to note that the collisional damping length along the magnetic field of the TG mode for the parameters of the Chen experiment, ${ }^{18} l_{c} \sim \omega_{c e} / \nu_{e i} k_{0 \mathrm{TG}}$, is approximately $l_{c} \approx 18 \mathrm{~cm}$, i.e., of the same order of value as the turbulent damping length $l_{T}$ of the helicon wave.

From Eq. (28) we can estimate the magnitude of the electric field strength of the strong ion-sound turbulence,

$$
E_{x} \sim \frac{m_{i} \omega_{s} \mathrm{v}_{\mathrm{Ti}}}{\mathrm{e}} \sqrt{\frac{2 W}{n_{0 i} T_{i}}} .
$$

For the experimental conditions of Ref. $18\left(\omega_{s} \approx 0.7 \omega_{0}, \omega_{0}\right.$ $\left.=1.2 \times 10^{8} \mathrm{~s}^{-1}, T_{e}=4 \mathrm{eV}, T_{e} / T_{i} \approx 20, W / n_{0 e} T_{e} \sim 0.05\right)$ we have $E_{x} \approx 0.5 \mathrm{kV} / \mathrm{cm}$. The electron velocity amplitude $u_{y}$ in such an electric field is comparable with electron thermal velocity $\mathrm{v}_{\mathrm{Te}}=8 \times 10^{7} \mathrm{~cm} / \mathrm{s}$. The motion of the electrons along the magnetic field is due to the action of the $E_{\|}$component of the ion-sound electrostatic electric field, equal to $E_{\|}$ $=E_{\perp}\left(k_{\|} / k_{\perp}\right) \sim 0.7 \mathrm{~V} / \mathrm{cm}$ for the experimental conditions of Ref. 18. Thus velocity of resonance electrons along the magnetic field is equal to $u_{e \|} \sim e E_{\|} / m_{e} \gamma_{e} \sim 4 \times 10^{7} \mathrm{~cm} / \mathrm{s}$, i.e., of the order of the electron thermal velocity $\mathrm{v}_{\mathrm{Te}}=8 \times 10^{7} \mathrm{~cm} / \mathrm{s}$. The velocity range for electrons that may be trapped by ionsound waves is $(\Delta \mathrm{v})_{\operatorname{Trap}} \sim\left(2 e E_{\|} / m_{e} k_{\|}\right)^{1 / 2}$ and for $k_{\|} \approx 0.3$ $\mathrm{cm}^{-1}$ is comparable with electron thermal velocity. These estimates show that the dynamics of electrons in helicon sources are strongly nonlinear and completely determined by electron "collisions" with ion-sound waves. So in spite of the weakness $(\gamma \ll \omega(k))$ of the kinetic parametric ion-sound instability considered, its ultimate nonlinear stage and the saturation are determined not by weak turbulence effects, but by the strong turbulence effects such as nonlinear resonance broadening. The effects of the weak turbulence are only significant during the initial development of the instability. 
In the case of strong ion-sound turbulence, the ion heating rate across the magnetic field is estimated from the ion renormalized quasilinear equation ${ }^{21,22}$ in which effect of the broadening of the resonances is included. The predicted ionheating rate is given by

$$
\frac{\partial T_{i \perp}}{\partial t} \sim \frac{1}{\tau_{i}} T_{i \perp} \sim \gamma_{e} \frac{W}{n_{0 e} T_{e}} \frac{T_{e}}{T_{i \perp}} T_{i \perp} .
$$

The principal difference between this equation and Eq. (26) is that this result is based on the ion renormalized quasilinear equation instead of Eq. (22). Equation (22) assumes saturation arises from the induced scattering of ion-sound waves by ions whereas Eq. (35) assumes that the saturated state arises from resonance broadening with the energy density $W$ in the saturated state given by Eq. (29).

Another important point is that the ion temperature anisotropy is not destroyed by ion-ion collisions when $\tau_{i \perp} \nu_{i i}$ $<1$, i.e., when the ion heating rate exceeds the ion-ion collision frequency. For the experiments of Chen et al. ${ }^{18}\left(T_{e}\right.$ $=4 \quad \mathrm{eV}, \quad T_{e} / T_{i} \approx 20, \quad \gamma \approx 2.3 \times 10^{6} \mathrm{~s}^{-1}, \quad \nu_{i i(\text { Argon })} \sim 4$ $\left.\times 10^{6} \mathrm{~s}^{-1}, W / n_{0 e} T_{e} \sim 0.05\right)$ we have $\tau_{i}^{-1} \sim 1.85 \times 10^{6} \mathrm{~s}^{-1}$ and $\tau_{i \perp} \nu_{i i} \approx 2$, i.e., ion heating anisotropy should not occur in the Chen experiments. However, for the argon helicon plasma experiments of Scime et al. ${ }^{12}$ in which significant ion temperature anisotropy was observed $\left(T_{e} \approx 5 \mathrm{eV}, T_{i \perp} \approx 0.4\right.$ $\mathrm{eV}, n_{0 i} \approx 1.4 \times 10^{12} \mathrm{~cm}^{-3}, B_{0}=800 \mathrm{G}, \omega_{0}=5 \times 10^{7} \mathrm{~s}^{-1}$ $\left.\approx \omega_{L H}\right)$ for $\left|\gamma_{\max }(k) / \omega(k)\right| \sim 0.3$ we have $W / n_{0 e} T_{e} \sim 0.065$, $\tau_{i} \sim 10^{-7} \mathrm{~s}, \quad \nu_{i i} \approx 7 \times 10^{5} \mathrm{~s}^{-1}$ and $\tau_{i \perp} \nu_{i i} \approx 0.07$. Therefore, ion temperature anisotropy is predicted by this model. Note, that the equation

$$
\ln ^{-1}\left[\frac{k \mathrm{v}_{s}}{\gamma_{e}}\left(\frac{\pi}{8}\right)^{1 / 2}\left(\frac{T_{e}}{T_{i}}\right)^{3 / 2}\right] \geqslant \frac{2 T_{i}}{T_{e}}
$$

defines a threshold condition for the amplitude of the helicon wave $E_{0} \sim E_{0 x}$ [because of the relationship $a$ $\approx c k_{y} E_{0 x} / \omega_{0} B_{0}$ in Eq. (4)] or for the ratio $T_{i} / T_{e}$ required for the excitation of the ion-sound kinetic parametric instability and subsequent turbulent plasma heating. The ion heating observations and these calculations suggest that the threshold condition of Eq. (31) is likely satisfied for the experiments of Scime et al.

\section{CONCLUSIONS}

In this work we have shown that ion heating measurements can provide important information about the role of nonlinear and turbulent processes in the operation of helicon plasma sources. We have shown that induced scattering of ions by short wavelength, spectrally anisotropic, ion-sound parametric turbulence can lead to anisotropic heating of ions predominantly across the magnetic field. The anisotropic ion heating can then result in nonlinear resonance broadening. Anisotropic ion heating is observed experimentally and may provide confirmation of the importance of parametrically driven plasma turbulence in the absorption of the helicon pump wave and heating of plasma components in helicon plasma sources.

Another source for the excitation of parametric ionsound turbulence is the Trivelpiece-Gould (TG) wave ex- cited by mode conversion from the helicon wave near the plasma surface..$^{3-5}$ Although the electric field strength of the TG wave significantly exceeds the value of $E_{0}$ for the helicon wave, the ion-sound turbulence excited by the TG wave is not responsible for the anomalous plasma heating and absorption of the TG wave. Observations of long wavelength ion-sound waves excited by the TG mode may provide indirect evidence of the existence of shorter wavelength ionsound waves believed to be responsible for the anomalous electron and ion heating and the anomalous absorption of the helicon waves since both sets of waves are excited at the same plasma conditions. In spite of the weakness $[\gamma$ $\gg \omega(k)]$ of the kinetic parametric ion-sound instability considered, the ultimate nonlinear stage and the saturation are determined not by weak turbulence effects, but by the effects of the strong turbulence. Estimates show that the motion of electrons and ions in such turbulence becomes strongly nonlinear with velocities larger then the their respective thermal velocities and is completely determined by their interaction with the ion sound turbulence. Therefore, the development of a complete theory of strong ion-sound parametric turbulence is of prime importance for understanding the operation of helicon plasma sources.

We also showed that the values of $T_{i}$ and $T_{e}$ (as well as the magnitude of the ratio $T_{e} / T_{i}$ ) are critically important for the development of the kinetic ion-sound parametric instability and turbulent plasma heating. For the stationary state of a continuously operated helicon source, the absolute values of $T_{i}$ and $T_{e}$ are determined by the coupled equations for ion and electron temperatures that depend on ion and electron heating and cooling due to thermal conductivity, charge exchange collisions, and radiation.

\section{ACKNOWLEDGMENTS}

Work at WVU was performed with support from National Science Foundation Grant No. ATM-99988450 and the U.S. Department of Energy under Grant No. DE-FG0297ER54420.

${ }^{1}$ R. W. Boswell, Plasma Phys. Controlled Fusion 26, 1147 (1984)

${ }^{2}$ F. F. Chen and D. D. Blackwell, Phys. Rev. Lett. 82, 2677 (1999).

${ }^{3}$ P. K. Shamrai and V. P. Taranov, Plasma Phys. Controlled Fusion 36, 1719 (1994).

${ }^{4}$ P. F. Vorobyov and A. A. Rukhadze, Fiz. Plazmy 20, 1065 (1994).

${ }^{5}$ B. Fisher, M. Kramer, and Th. Enk, Plasma Phys. Controlled Fusion 36, 2003 (1994).

${ }^{6}$ A. I. Akhiezer, V. S. Mikhailenko, and K. N. Stepanov, Phys. Lett. A 245, 117 (1998).

${ }^{7}$ N. M. Kaganskaya, M. Kramer, and V. L. Selenin, Phys. Plasmas 8, 4694 (2001).

${ }^{8}$ M. Kramer, B. Lorenz, B. Clarenbach, and V. L. Selenin, Europhysics Conference Abstracts Vol. 26B, 29th European Physical Society Conference on Plasma Physics and Controlled Fusion, Montreux, 17-21 July 2002, edited by R. Behn and C. Varandas, CD ROM edition (European Physical Society, Paris, 2002), p. O-4.08.

${ }^{9}$ J. Kline, E. Scime, R. Boivin, A. M. Keesee, X. Sun, and V. Mikhailenko, Phys. Rev. Lett. 88, 195002 (2002).

${ }^{10}$ J. L. Kline and E. Scime, Phys. Plasmas 10, 135 (2003).

${ }^{11}$ V. F. Virko, G. S. Kirichenko, and K. P. Shamray, Book of Abstracts of International Conference and School on Plasma Physics and Controlled Fusion held in Alushta (Crimea), Ukraine, 16-21 September 2002. Published by National Science Center "Kharkov Institute of Physics and Technology," 2002, I-21, p. 22. 
${ }^{12}$ E. E. Scime, P. A. Keiter, M. W. Zintl, M. M. Balkey, J. L. Kline, and M. E. Koepke, Plasma Sources Sci. Technol. 7, 186 (1998).

${ }^{13}$ M. M. Balkey, R. Boivin, J. L. Kline, and E. E. Scime, Plasma Sources Sci. Technol. 10, 284 (2001).

${ }^{14}$ J. L. Kline, E. Scime, R. Boivin, A. M. Keesee, and X. Sun, Plasma Sources Sci. Technol. 11, 413 (2002).

${ }^{15}$ V. S. Mikhailenko, V. V. Ol'shansky, and K. N. Stepanov, Problems of Atomic Science and Technology, edited by National Science Center "Kharkov Institute of Physics and Technology," 1, 43 (2000) (ISSN 15626016).

${ }^{16}$ B. B. Kadomtsev, Plasma Turbulence (Academic, London, 1965).

${ }^{17}$ A. I. Akhiezer, V. S. Mikhailenko, V. V. Ol'shansky, and K. N. Stepanov,
Plasma Phys. Rep. 26, 575 (2000).

${ }^{18}$ F. F. Chen, I. D. Sudit, and M. Light, Plasma Sources Sci. Technol. 5, 173 (1996).

${ }^{19}$ V. S. Mikhailenko and K. N. Stepanov, Bull. Am. Phys. Soc. 45, 134 (2000); Proceedings of the 42nd Annual Meeting of the Division of Plasma Physics and the 10th International Congress on Plasma Physics, 23-27 October 2000, Quebec City, Quebec, Canada (ICPP Books of Proceedings), Vol. 1, p. 61.

${ }^{20}$ L. M. Kovrizhnykh, Sov. Phys. JETP 22, 1014 (1965).

${ }^{21}$ A. M. Sleeper, J. Weinstock, and B. Bezzerides, Phys. Fluids 16, 1508 (1973).

${ }^{22}$ T. H. Dupree, Phys. Fluids 9, 1773 (1966). 